\title{
Heterogeneity and increasing returns may drive socio-economic transitions
}

\author{
Gérard Weisbuch • Vincent Buskens • Luat Vuong
}

Published online: 26 September 2008

(C) The Author(s) 2008. This article is published with open access at Springerlink.com

\begin{abstract}
There are clear benefits associated with a particular consumer choice for many current markets. For example, as we consider here, some products might carry environmental or 'green' benefits. Some consumers might value these benefits while others do not. However, as evidenced by myriad failed attempts of environmental products to maintain even a niche market, such benefits do not necessarily outweigh the extra purchasing cost. The question we pose is, how can such an initially economically-disadvantaged green product evolve to hold the greater share of the market? We present a simple mathematical model for the dynamics of product competition in a heterogeneous consumer population. Our model preassigns a hierarchy to the products, which designates the consumer choice when prices are comparable, while prices are dynamically rescaled to reflect increasing returns to scale. Our approach allows us to model many scenarios of technology substitution and provides a method for generalizing market forces. With this model, we begin to forecast irreversible trends associated with consumer dynamics as well as policies that could be made to influence transitions.
\end{abstract}

Keywords Market competition · Willingness to pay · Transitions · Heterogeneity

\footnotetext{
G. Weisbuch $(\bowtie)$

Laboratoire de Physique Statistique (Laboratoire associé au CNRS (UMR 8550), à l'ENS et aux Universités Paris 6 et Paris 7), de l'Ecole Normale Supérieure, 24 rue Lhomond, 75231

Paris Cedex 5, France

e-mail: weisbuch@1ps.ens.fr

V. Buskens

Department of Sociology, Utrecht University, Heidelberglaan 2, 3584 CS, Utrecht, The Netherlands e-mail: v.buskens@uu.nl

L. Vuong

School of Applied and Engineering Physics, Cornell University, Ithaca, NY 14853, USA
} 


\section{Introduction}

The adoption of 'green' technology is hard to predict because it implies many intertwined social and economic factors plus retro-action loops. Because even a partial description of these factors and their interaction seem intricate, authors are attracted by the multi-agent modeling approach (see, e.g., Bousquet et al. 2001 and the related special issue of JASSS). This multi-agent approach suffers from numerous limitations when interpreting the impact of model parameters, and does not provide insight for rigorous comparison between models and real systems. Moreover, model predictions may abruptly change within very small regions of the parameter space (see also Weisbuch 2000) and these dynamics are both easy to overlook and difficult to investigate with agent-based models.

We here propose a simple, soluble model that addresses the intricate nature of the market (heterogeneity in consumer responses, social influence, and increasing returns to scale of production prices) and generalize various parameters that would otherwise lead to unwieldy complexity in simulation (see also Kemp 1998 for a related model, but without increasing returns to scale). Our increasing returns to scale includes both economic effects related to resources and supply, as well as social influences, i.e., increased demand that arises via peer pressure from those who already own the product, which is an argument already made by Brian Arthur (1994). To analyze the competition between goods with different prices and varied quality or environmental features, we subsume the impact of different cultures, social influences, government policy, and advertising into a single curve, which we define as the 'willingness to pay' (WTP) function. WTP illuminates the consumer population distribution as a function of price and describes how people vary in the extent to which they want to pay for environmental benefits. We assume that the normalized WTP distribution has a fixed shape, while the price associated with each market product varies with its market share.

Although the model turns out to have nice implications, this is not the only aim of this paper. We also want to illustrate the KISS (Keep It Simple Stupid !!) approach to complex systems. The KISS acronym is not much more than a popular version of a much older principle in the philosophy of sciences that claims simple theories are often preferable "because their empirical content is greater; and because they are better testable" as Popper $(1992,128)$ put it. In this sense, our approach to transitions is complementary to more conceptual as well as agent-based modeling approaches that lack the strict focus on simplicity.

In our view, the more conceptual approaches (for some recent work as well as many references to related older work, see Geels 2007; Geels and Schot 2007) are very useful for obtaining a framework in which many transitions can be both described and analyzed at a conceptual and descriptive level. In this framework, transitions can be placed with real life complexity including aspects such as technologies, impact factors, beliefs, social influence, economic factors, the role of political agencies, etc. For Geels (2007), this approach enabled the detailed understanding of the specific case of the Dutch Highway system. However, to have predictive power in such a framework should also be falsifiable. In the general informal setup as it is built up, e.g., by Geels and Schot (2007), we think every transition could be placed within 
the system and we see no possibilities to falsify it as a theory. Formalizing a complex framework, as described above in an agent-based model, requires so many parameters (see, e.g., Chiong, Meza, and Yãcel (2008) in this volume) that it is almost impossible to obtain a comprehensive analysis of the complete parameter space.

In the KISS approach, we start from the other side. Although we realize that a simple model cannot cover all the complexity of every single case, we are not prepared to add explicit complexity to the model as long as we do not have evidence that adding explicit complexity via additional parameters could uncover new patterns in transitions or enable the prediction of general trends. To connect our terminology with that currently used in the transition literature, we begin with a simple utility maximizing actors at the micro level and a very simple operationalization of the macro-level landscape allowing for only three different technologies to emerge into different mixtures of diffusion (say, regimes). Clearly, this approach is closely related to Coleman's (1990, chapter 1) micro-macro scheme used for building social science theories. By gradually adding complexity (see also Lindenberg 1992a), we gain insight to realistic trends, and achieve qualitative or semi-quantitative predictions on characterization of dynamical regimes and their transitions (see Sects. 3.2 and 3.3 of the present paper).

In this paper, we add two important elements of complexity to Coleman's model, namely, we provide actors that are not only interested in money but make decisions based on environmental benefits and the actors that we employ are heterogeneous in this respect. In addition, we do not just incorporate two competing technologies, but three technologies. This enables us to formalize the complete system in terms of just four crucial parameters and to investigate the full parameter space.

One additional advantage of this simple formalization in mathematical terms is that some of the dynamics can be interpreted in terms of different processes in real life; for instance:

- Positive return to scale can be interpreted either as a decrease in production cost or an externality making more attractive a product bought by many people;

- A model with a WTP distribution as we develop below is equivalent to a probabilistic model with a probability to buy depending upon the difference between the actual price and WTP (see Nadal et al. 2005).

The formalization also enables robust inspection of our results with respect to specific assumptions in the model. For example, as we will demonstrate below, the fact that the same dynamical regimes is displayed in a large region of the parameter space can be generalized to variations in the form of the functions used in the model, in analogy with what physicists call universality. Both the functional form of the return to scale function (provided that price is monotonically decreasing as a function of the market share) and the WTP distribution (provided that it is single-peaked) can be changed while maintaining the same dynamical regime.

In conclusion, the KISS approach manages the complexity of the real world and our incomplete characterization of local processes to obtain robust predictions and provide insight in the global consequences of local processes. We will now illustrate this in our example of the competition between technologies with different environmental benefits.

This paper is organized as follows. Section 2 describes our computational model with equations. Section 3 describes our results. We show that environmental technolo- 
gies will take over when a significant fraction of agents have already a WTP to pay for the green car whatever its market share and when the increasing returns coefficient is large (because of strong social effects or large production price reduction with production level). We also demonstrate how different conditions can lead to car 0 , 1 , or 2 dominating the market depending on the rescaling of price due to demand. Section 3.2 shows that our simple model not only provides reliable results but also analytic tools to evaluate multiple asymptotic solutions. For some parameter values, the dynamics have several attractors, which implies hysteresis effects. We extrapolate that the timing of the subsidies and grants at the immediate onset or emergence of a new technology may be crucial as trends are sometimes irreversible. Section 4 summarizes our conclusions and shows future directions for this model.

\section{A simple set of assumptions}

We start the description of our model with explaining in detail our assumptions. We use the example of more or less green cars below, but clearly any product for which there is some non-monetary benefit related to the product can be used instead. So where we write for convenience 'car' below, one could read 'product,' and when we write environmental benefits or 'greenness,' one could read other non-monetary benefits (amenities).

- Consumers care about two aspects of a car, i.e., the price and 'greenness' of the car;

- Environmentally superior technologies are more expensive (given a similar market share);

- Cars with a larger market share are cheaper;

- People vary in their extent to which they want to pay for environmental benefits, i.e., heterogeneity of WTP (see Gordon et al. 2005, 2007; Nadal et al. 2005).

- People choose the alternative they prefer.

The second assumption is more for convenience than that it is really necessary. Environmentally inferior technologies that are more expensive would not be chosen by anybody, because given that there are two products with prices below what a consumer is willing to pay, this consumer chooses the 'greener' technology. Thus, given the first assumption, products that are more expensive and less 'green' cannot survive in this market anyway.

The third assumption has two possible interpretations. A larger market share has advantages of scale for the producers, which implies that cars can be produced for a lower price when the market share is larger. From the buyers perspective, social influence effects affect the utility consumers get from a product: a more popular car is more attractive for most individuals than a car that nobody bought, which changes the specific willingness to pay for that type of car. But this is equivalent to saying that the price of that type of car decreases. Since only the difference between WTP and prices is relevant to buyers' decisions, both effects have the same results (Arthur 1994). In theory one should make a distinction between the production costs which are influenced by market shares say during the actual year, and the social influence 


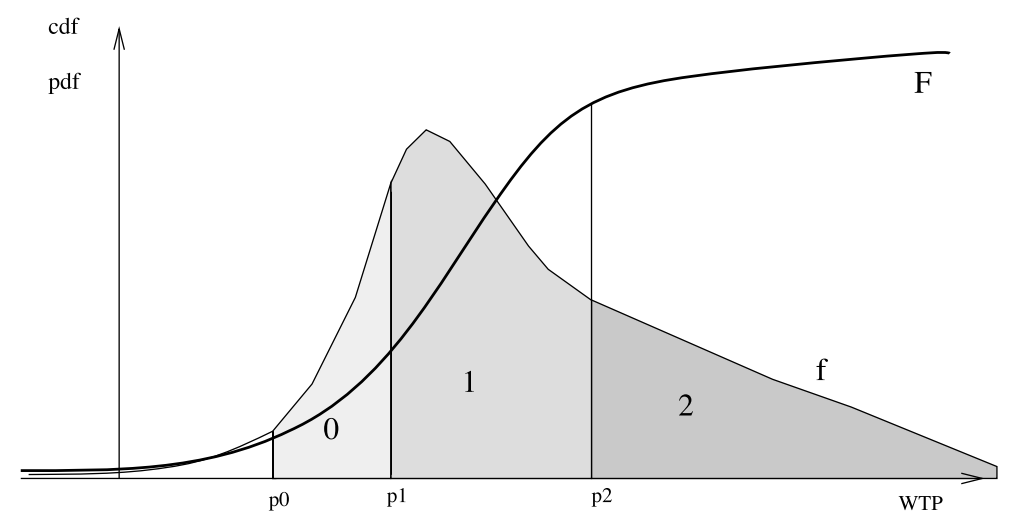

Fig. 1 Distribution of willingness to pay (WTP) and market shares. Consumers with WTP larger than $p_{i}$ may choose car $i$ with market share $u_{i}$ equal to the shaded area

terms which takes into account how many cars of each type have been bought in the past (the integrated yearly market share). In practice, because of the irreversibility of the capital investment in the automobile industry, the actual production cost also integrate investments and the two effects are driven by the integrated market share.

There are three technological options: standard (0), intermediate (1), 'green' (2).

- Each option $i$ has its own maximum cost $P_{0 i}$, which equals the cost at a zero market share;

- All options have the same linear returns to scale coefficient $k$ (i.e., $p_{i}=P_{0 i}-k u_{i}$, where $p_{i}$ and $u_{i}$ are the actual price and market share of product $i$, respectively);

- There is one distribution of WTP for environmental benefits (e.g., uniform or bellshaped)

It seems a rather strong assumption that all products experience a similar of linear decreasing returns to scale. However, this assumption could very easily be relaxed in future extensions of the model and is not crucial for the implications we derive in this paper.

Figure 1 shows an arbitrary distribution of WTP. Consumers with WTP larger than $p_{i}$ may choose car $i$ with market share $u_{i}$ equal to the shaded area. More specifically, consumers with WTP larger than $p_{2}$ choose car 2. Of the remaining market share, consumers with WTP larger than $p_{1}$ choose car 1 . Finally, from the still remaining market share, consumers with WTP larger than $p_{0}$ choose car 0 . According to the WTP distribution, some agents might decide to buy no car so that $u_{0}+u_{1}+u_{2} \leq 1$.

In the following subsection, we explain how the stable market shares are computed.

\subsection{Equations}

Given that we assume the same returns to scale, the price of a product follows a linear return to scale function. 


$$
p_{i}=P_{0 i}-k u_{i}
$$

Because of the influence of market share on actual prices, the order among actual prices $p_{i}$ may differ from maximum prices at zero market share. A first operation is to order products $i$. New indices $j$ are used for prices ordered by increasing actual prices, according to market share distribution. Some products might lose their rank: in such a case, we consider that every time a product $j$ has larger price $p_{j}$ than a product with a better environmental quality, it disappears from the market: nobody is interested to buy a more expensive product with a lower environmental quality. With final indices $j$, equilibrium market shares obey:

$$
u_{j}=F\left(p_{j+1}\right)-F\left(p_{j}\right)
$$

where $F(p)$ is the cumulative WTP distribution. This simple set of equations is soluble, either directly for simple expressions of $F(p)$, as we do in Sect. 3.2 on results or through transcendental equations.

While these equations provide the (possible multiple) solutions for the stable proportions of each product in the market, one can also simulate the dynamics to these solution by assuming that at each point in time a certain proportion $\lambda$ of consumers is choosing a new car according to the prices and preferences at that point in time. The related dynamics of market shares is given by:

$$
u_{j}(t+1)=(1-\lambda) u_{j}(t)+\lambda\left(F\left(p_{j+1}, t\right)-F\left(p_{j}, t\right)\right)
$$

The simulations in this paper were done for two simple WTP distributions: the uniform distribution and a logit distribution. The corresponding equation for the cumulative distribution $F(p)$ is piecewise linear for the uniform distribution:

$$
F(p)=\frac{p-p_{m}}{p_{M}-p_{m}}
$$

between the minimum $p_{m}$ and the maximum $p_{M}$ WTP price. Below $p_{m}, F(p)=0$, above $p_{M}, F(p)=1$.

For the bell-shaped cumulative distribution we use a logit expression:

$$
F(p)=\frac{1}{1+\exp (-\beta p)}
$$

where $\beta$ is inversely proportional to the width of the distribution. If we define the width as the inverse slope at the point of inflexion $(p=0)$, we obtain:

$$
w=\frac{4}{\beta} .
$$

The corresponding graphs appear in Fig. 2. 
Fig. 2 Distributions of willingness to pay (WTP). A uniform partial distribution function (pdf) and the corresponding cumulative distribution function (cdf) are drawn in black. The corresponding logit distributions are drawn in gray (bell-shaped for the pdf, S-shaped for the cdf)

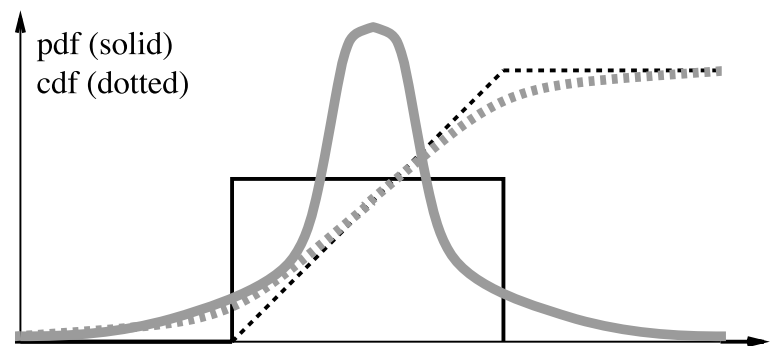

$\mathrm{p}$

\section{Results}

\subsection{Time evolution of market shares}

The evolution of market shares for the three products is easily simulated for a fixed set of parameters. The two plots of Fig. 3 only differ by the value of the maximum price of the green product $P_{02}$. In both cases, as in most simulations, only the standard product is initially present $\left(u_{0}(t=0)=1, u_{1}(0)=0, u_{2}(0)=0\right)$. This corresponds with the assumption that we want to predict at which maximum prices it is possible to enter the market for greener products. In other words, we want to see under which conditions green products that initially occupy a niche can become the main regime and under which conditions this is less likely.

Asymptotic market shares are reached in a few characteristic times $(\lambda)^{-1}$, where $(\lambda)^{-1}$ corresponds to a minimum characteristic time of evolution towards equilibrium.

The connection between the evolution of market shares and prices is evident from Fig. 4, obtained for the same parameter values as the right plot of Fig. 3. The initial increase of $u_{1}$ and $u_{2}$ decreases $u_{0}, p_{1}$, and $p_{2}$, and increases $p_{0}$. Since $p_{1}$ decreases slower than $p_{2}, u_{1}$ saturates after an initial increase and finally decreases. It is also clear that due to the lower $P_{02}$ in the right plot, the green car becomes the dominant product in the market and completely drives out again the intermediate car, while the standard car maintains a minority share in the market.

Because of the increase of $p_{0}$, some consumers do not find any car to buy (unless $k$ is larger than the width of the distribution, see further Sect. 3.3). The asymptotic sum of market shares is then less than one. Such a situation is often encountered.

\subsection{Dynamical regimes}

To obtain a more complete overview about how the parameters affect the outcomes of the dynamics, we will now provide a more complete overview of possible end points of the dynamics, the so-called 'dynamical regimes.' The parameters are a priori:

- Two parameters defining the center of the WTP distribution and its width (we only study symmetric distributions).

- The three maximum prices $P_{0 i}$.

- The slope $k$ of the increasing returns expression. 

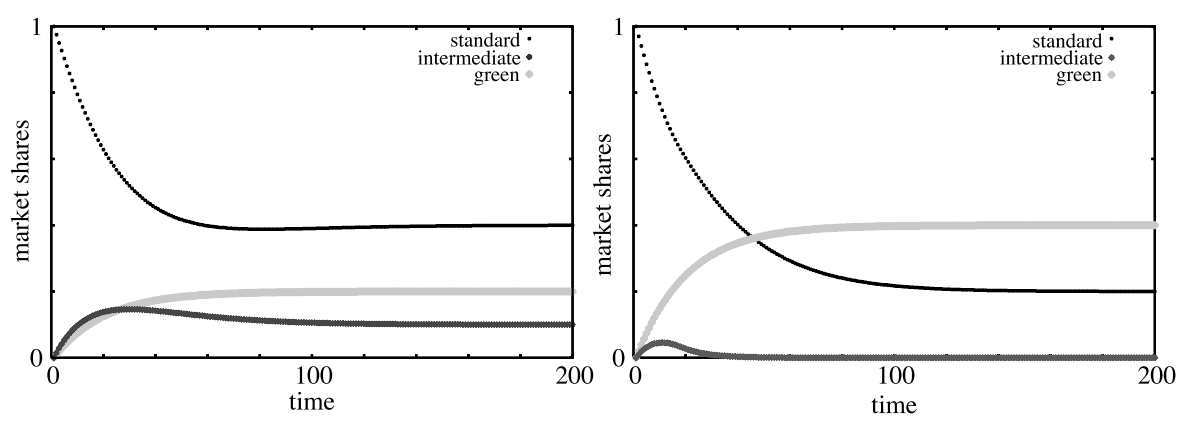

Fig. 3 Time evolutions of market shares: standard (black), intermediate (dark gray), and green (light gray) products. $(\lambda)^{-1}=10$

Fig. 4 Time evolutions of market shares and prices

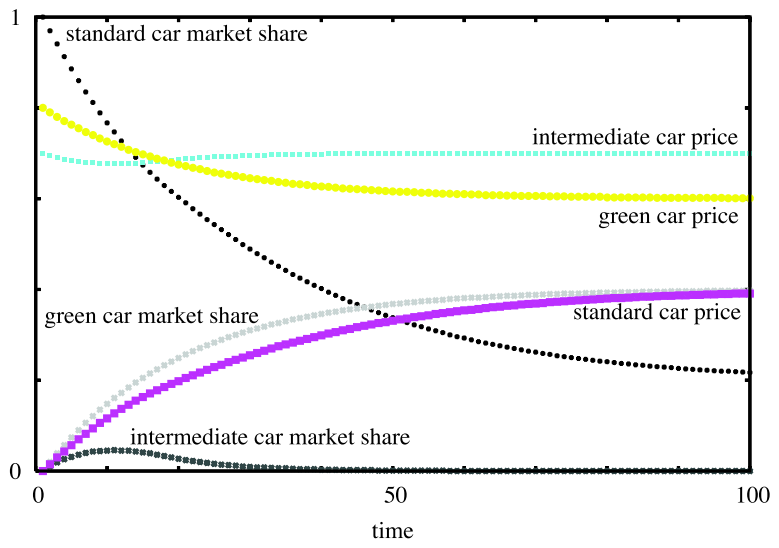

They in fact reduce to four independent parameters, because only the relative position of prices with respect to the WTP distribution is important. We will find that only the ratio of $k$ to the width of the WTP distributions plays a role for simple distributions.

One should realize that $\lambda$ is a kinetic parameter that only influences how fast attractors are reached, but not the attractors themselves.

Figures 5 to 7 were made for constant maximum prices of standard and green product, and for fixed WTP. We sometimes used uniform, and sometimes the logit distribution. The two varying parameters are then $k$ and $P_{01}$. This turns out to provide already a quite complete overview of which dynamical regimes occur and how they depend on the parameters.

For the above choice of parameters, whether the green product becomes dominant depends essentially from how far $P_{02}$ is from the maximum WTP: in other words what is the potential market share taking only into account the maximum price of the green product. Of course, $u_{2}$ increases with $k$. In fact, $u_{2}$ is independent of $P_{01}$ and $P_{00}$. At equilibrium it always obeys:

$$
u_{2}=1-F\left(P_{02}-k u_{2}\right)
$$


Fig. 5 Asymptotic market shares as of function of $k$ and $P_{01}$. Uniform WTP distribution $[0,1], P_{00}=0.5, P_{02}=0.8 . k$ varies between 0 (no return to scale) and 0.5. $P_{01}$ varies between $P_{00}$ and $P_{02}$. Light gray stripes, dark gray dots, and black lines correspond to the market share of the green, intermediate, and standard products, respectively

Fig. 6 Asymptotic market shares as of function of $k$ and $P_{01}$. Uniform WTP distribution $[0,0.9]$. All other items as in Fig. 5
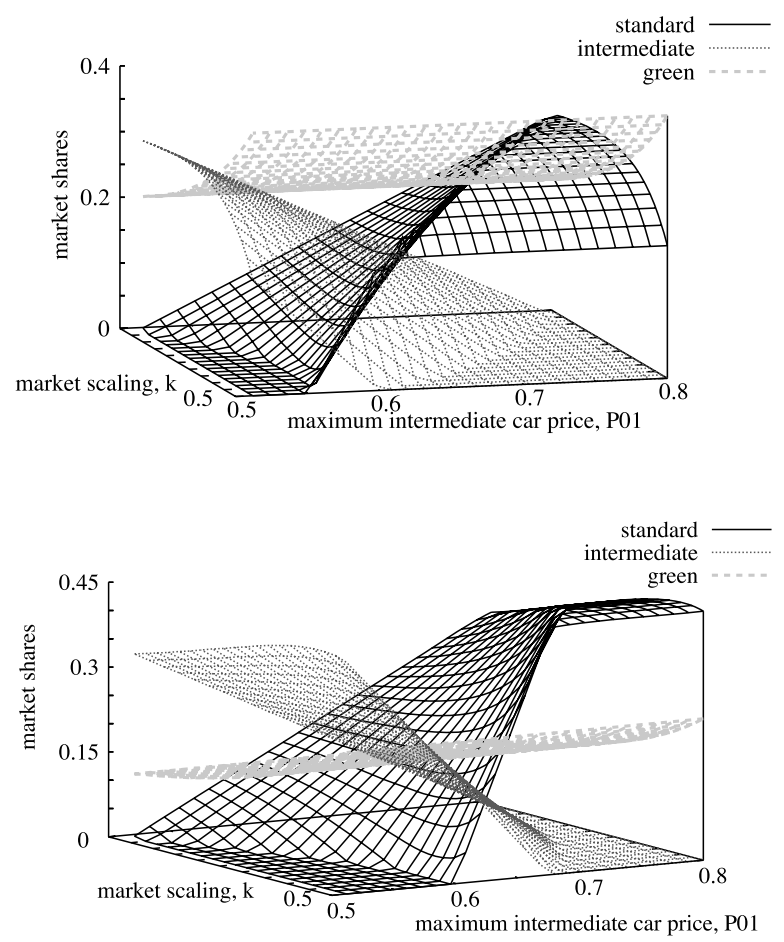

Fig. 7 Asymptotic market shares as of function of $k$ and $P_{01}$. Uniform WTP distribution $[0,1.1]$. All other items as in the Fig. 5

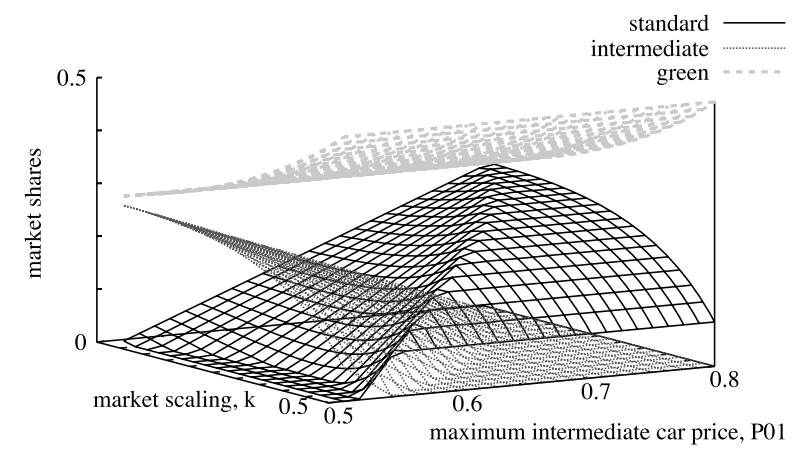

since the 'green' product is always chosen when the customer's WTP is larger than $p_{2}$.

For the uniform WTP distribution this gives:

$$
u_{2}=\frac{p_{M}-P_{02}}{p_{M}-p_{m}-k}
$$

where $p_{M}$ and $p_{m}$ are respectively the upper and lower bound of the WTP distribution. This expression is only valid for $k<w$ (check Sect. 3.3 for the opposite case). In addition, $p_{M}$ should be larger than $P_{02}$ otherwise $u_{2}=0$. It shows that the mar- 
ket share of the green car only depends upon two reduced parameters, $\frac{p_{M}-P_{02}}{p_{M}-p_{m}}$ and $\frac{k}{p_{M}-p_{m}}$.

The competition between the standard and intermediate car, on the other hand, mostly depends upon $P_{01}$ : the standard car is favored when $P_{01}$ is close to $P_{02}$, and the intermediate car when $P_{01}$ is close to $P_{00}$.

For the uniform WTP distribution, (2) in $u_{2}, u_{1}$, and $u_{0}$ are easily solved; the expressions for $u_{2}, u_{1}$, and $u_{0}$ depend of the ranking of prices. In the case of $p_{0} \leq$ $p_{1} \leq p_{2}$, they are written in the simple case of a $[0,1]$ WTP distribution:

$$
\begin{aligned}
& u_{2}=\frac{1-P_{02}}{1-k}, \\
& u_{1}=\frac{p_{2}-P_{01}}{1-k}, \\
& u_{0}=\frac{p_{1}-P_{00}}{1-k}
\end{aligned}
$$

where actual prices $p_{2}$ and $p_{1}$ are obtained from the corresponding values of $u_{i}$. One sees from these equations that markets shares are zero (and the equations have to be re-ordered) whenever $p_{i+1} \leq P_{0 i}$. These conditions re-written in terms of initial parameters are:

$$
\begin{aligned}
& u_{1}=0 \quad \text { iff } P_{01} \geq \frac{P_{02}-k}{1-k} \\
& u_{0}=0 \quad \text { iff either } P_{00} \geq \frac{P_{01}}{1-k}-\frac{k\left(P_{02}-k\right)}{(1-k)^{2}} \text { and } P_{01}<\frac{P_{02}-k}{1-k},
\end{aligned}
$$

or

$$
P_{00} \geq \frac{P_{02}-k}{1-k} \text { and } P_{01} \geq \frac{P_{02}-k}{1-k}
$$

For more general distributions, e.g., the logit distribution, a similar iterative procedure yields market shares and prices, but the equations are transcendental rather than explicitly soluble.

\subsection{Hysteresis}

Equations such as (2) are well-known in physics, e.g., in the Mean Field theory of ferromagnetism (Kittel and Kroemer 1980) and to some extent in economics (Föllmer 1974; Weisbuch et al. 2000), in the case of increasing returns or social influence. They are known to produce 'phase' or 'regime' transitions when the number of their solutions goes from one to three as a function of a parameter which is $k / w$ in our case (for the logit distribution $w=4 / \beta$ ).

Figure 8 allows to easily understand the regime transition. It is a graphical solution of (2) rewritten as:

$$
1-u=F(p-k u)
$$


Fig. 8 Graphical solution of (2) rewritten as $1-u=F(p-k u)$. Abscissa is $x=p-k u$, and ordinate $1-u$. The $F(x)$ curve (cdf), represented in gray for the logit distribution of WTP and in black for a uniform distribution, intersects dotted black lines $1-u$ in one or three points according to the value of $k$ for the particular choice of $p$. $k_{m} \leq 1 \leq k_{M}$

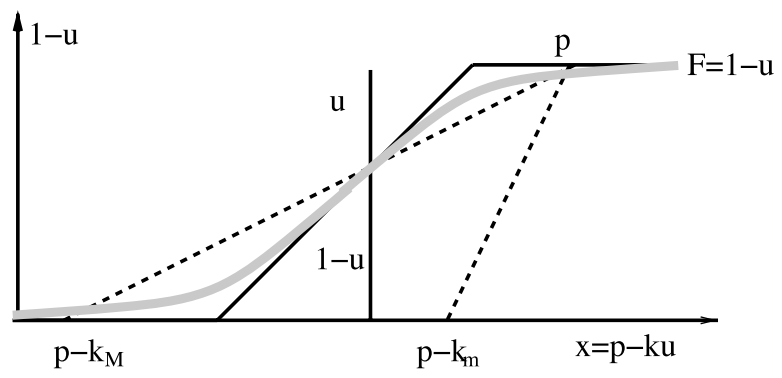

The curves corresponding to the two sides of the equation are drawn in the plan ( $p-$ $k u, 1-u)$. The grey and black solid curves correspond to $F(p-k u)$ (respectively, logit and uniform distributions). The dotted lines correspond to the left hand side $1-u$ as a function of $p-k u$; it is a straight line, which abscissa is $p$ when $u=0$ and $p-k$ when $u=1$. Two dotted lines are drawn corresponding to the two cases of a large $k, k_{M}$ and a small $k, k_{m}$.

Three solutions may be obtained if the slope of the straight line $1-u$ is lower than the largest slope of the $F$ function. This slope is $k / w$ for the uniform distribution and $4 k / \beta$ for the logit distribution. The corresponding conditions for $k$ versus the WTP distribution parameters are thus written:

$$
\begin{aligned}
& k \geq w, \\
& k \geq \frac{4}{\beta}
\end{aligned}
$$

When $k$ is above the threshold, one or three fixed points are then obtained depending upon the value of the maximum price. In the case of three fixed points, the central one is unstable but the two extreme are attractors; which attractor is actually reached depends upon initial conditions: large $u$ values attractor is, e.g., obtained when initially $u(0)=1$ (and small $u$ attractor when $u(0)=0$ ). The central fixed point separates the two attractor regions.

We considered until now $P_{02}$ as a fixed parameter. Let us now discuss the effect of variations of $P_{02}$, which could be due to technological progress or to strategic moves of the producer of car 2, or to government subsidies etc. Figures 9 and 10 illustrate the hysteresis cycle when $P_{02}$ is varied, and the transition between the two regimes when both $k$ and $P_{02}$ are varied. They were drawn by superimposing asymptotic simulation results obtained when initial conditions were first $u_{2}(0)=1$ (black curves) and then when $u_{2}(0)=0$ (gray curves). In the first case, we thus start without standard or intermediate cars. In the second case, we start with only standard cars. For parameters such that only one attractor exists, only the gray curves are visible. But when two are possible, the black curves are visible and correspond to $u_{2}(0)=1$.

The vertical transitions obtained in the parameter space for the attractor at specific values of $P_{02}$ still correspond to finite time dynamics with a characteristic time of $\lambda^{-1}$. 
Fig. 9 A hysteresis cycle obtained for $u_{2}$ when $P_{02}$ is varied. The black curve is $u_{2}$ for $u_{2}(0)=1$ and the gray curve when $u_{2}(0)=0$. The increasing returns coefficient $k=2$ is larger than the WTP distribution width $w=4 / \beta=1$. The two curves coincide when the price $P_{02}$ is either small or large. They differ in the intermediate price region

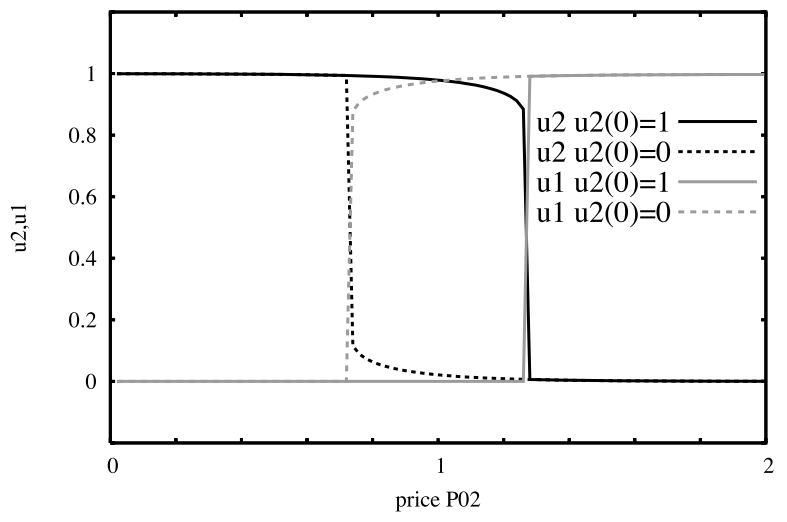

Fig. 10 Transition between the hysteresis regime and the one attractor regime when $k=\frac{4}{\beta}$. The green dotted sheet is obtained when $u_{2}(0)=1$ and the black sheet when $u_{2}(0)=0$. They differ only in the large $k$ and intermediate $P_{02}$ region

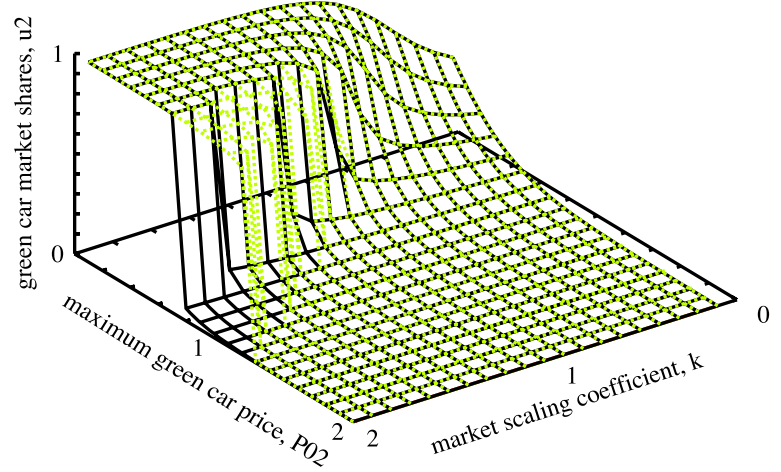

The middle part of Fig. 9 can be interpreted as follows. If we have a market that is completely dominated by green cars, the maximum price $P_{02}$ has to rise almost up to 1.3 before the market is taken over by the intermediate or standard car. On the opposite, if the green car does not have a substantial share of the market, the price of the green car has to drop almost to 0.7 before the green car can take over the market.

At the middle of hysteresis loop, $P_{02}$ is one width above the center of the WTP distribution. But since $k=2$, the actual price when $u_{2}=1$ is one width below the center of the distribution. Large values of $k$ imply a strong social influence with respect to price differences: varying $u_{2}$ between 0 and 1 scans a large percentage of the WTP distribution (96 percent). At the transition, $k=\frac{4}{\beta}=1$, this figure is already 63 percent.

We only discussed until now the dynamics of $u_{2}$ as a function of $k$ and $P_{02}$. Which of the two other products dominates or how they share the market when $u_{2}=0$ depends upon the actual values of $P_{00}$ and $P_{01}$.

Why should we care about hysteresis? After all, our starting assumption was that the intermediate and the green options are introduced after the standard option implying $u_{0}=1, u_{1}=u_{2}=0$ as initial conditions. Since there are several attractors in this regime, the issue of the adoption regime is especially sensitive to the parameter set-up: if we now consider that parameters can be under the influence of decision makers such as producers or government agencies, or exogenous events (e.g., oil 
prices, technical advances), the hysteresis regime can bring huge consequences for small parameter changes. For instance,

- If price $P_{02}$ is lower under the action of producers, advertising in the media, or government subsidies, a transition from the $u_{2} \simeq 0$ attractor to the $u_{2} \simeq 1$ attractor can be induced.

- Such an action does not have to be permanent: it might suffice to bring $u_{2}$ above the separatrix, the central fixed point, to bring the system in the basin of attraction of high $u_{2}$.

- Competitors might also have equivalent strategies.

- Sharp transition can be induced in this region through advertising by decision makers, thus changing effective prices for some of the products or the complete WTP distribution.

Multiple attractors are a challenge for scientists, but they are opportunities for decision makers. Of course, parameter changes in the single attractor regime also influence the outcome of the dynamics, but their influence is far less dramatic. Moreover, these effects are reversed as the parameter changes are undone, while the changes under multiple attractors might remain after parameter changes are undone.

\section{Conclusions}

In this paper, we have illustrated, how one can obtain interpretable but already quite complex dynamics from a simple model on the competition between more or less green technologies assuming that consumers have heterogeneous preferences over these goods. In the first part of the paper we have shown situations in which there was only one attractor for the dynamics. These dynamics show that the prices of the greener alternatives needs to be far enough from the boundary of the willingness to pay distribution to take over the market, and that the advantages of scale should be large enough to overcome price differences over time. Depending on the precise parameters, several different regimes are possible, differing in the number of equilibrium technologies: 1,2 , or 3 . In some situations, green technologies take over only a smaller part of the market, while the standard technology remains dominant. But there are also situations were first the intermediate technology conquers some of the market, and thereafter the most green technology gains some market share and takes most of the market.

Simulations were done with parameters being constant, by definition. One can also infer the results of technological or attitude changes over market shares dynamics. In situations in which there is basically only one attractor, temporary policy measure will not have permanent results because the process will reverse as soon as the policy measure ends.

In the second part of the results, we show that there are also situations in which there are multiple attractors of the dynamics. They typically occur when the width of the willingness to pay distribution is less than the increasing returns coefficient. As long as the market share of the products is either very small (or very high) the market is stable. However, if some agency is able to raise for some time the fraction 
of consumers using green cars, or if it could boost for some period the environmental consciousness of enough consumers, the system might jump to the situation in which most people drive in green cars. Such temporary policy measures could then have a stable result even if the measure is only temporary. Of course producers of standard cars could think of measures to get back to the first situation, but these would at least be quite costly for them.

Let us stress that these dynamic properties are generic: they do not depend upon a specific choice of the WTP distribution nor of the increasing return price function. They apply to any S-shape WTP cumulative distribution and any monotonic increasing return function.

Another way to test the robustness of a model is to study a probabilistic variant. Would the observed dynamical behavior be conserved in the presence of a random noise, a possible representation of other factors that we have not been able to take into account in our model? In fact, Nadal et al. (2005) have discussed the role of noise in buyers decisions. They have shown that a model with fixed WTP distribution, called a frozen disorder by physicists is equivalent to a model with single valued WTP, but where the decisions taken by the agents are probabilistic. In other words the agent would choose between different choices with a probability function of the different prices. The probability function chosen by economists is generally the logit function written as:

$$
\mathcal{P}(i)=\frac{\exp \left(-\beta p_{i}\right)}{\sum \exp \left(-\beta p_{j}\right)}
$$

giving the probability of choice $i$ among all other possible choices $j$.

The two models are mathematically equivalent and the market shares obey the same equations, provided that the distributions are the same. We infer from this that a model with both WTP and probability distributions corresponds to the same mathematical expression as in (2) with a distribution which is the convolution product of the original distributions.

Many extensions of the present model are possible, some more application specific, others including coupling with pollution and opinion dynamics, the role of government agencies etc. Some of these extensions might necessitate heavier simulation tools such as multi-agent systems. But anyway, the simple analysis that has been performed here already allows to figure out the influence of the parameters on the observed dynamical regimes and the level of behavioral complexity that can be expected for the heterogeneity of agents and increasing return hypotheses.

Acknowledgements We thank Jean-Pierre Nadal for illuminating discussions and the participants of the CMAST07 workshop in Leiden (Feb-March 2007). Gérard Weisbuch was also supported by E2C2 NEST 012410 EC grant. Vincent Buskens was supported by the UU-High Potential Program 'Dynamics of Cooperation, Networks, and Institutions.'

Open Access This article is distributed under the terms of the Creative Commons Attribution Noncommercial License which permits any noncommercial use, distribution, and reproduction in any medium, provided the original author(s) and source are credited. 


\section{References}

Arthur B (1994) Increasing returns and path dependence in the economy. University of Michigan Press, Ann Arbor

Bousquet F, Lifran R, Tidball M, Thoyer S, Antona M (2001) Agent-based modelling, game theory, and natural resource management issues. J Artif Soc Soc Simul 4(2). Available at http://www.soc.surrey. ac.uk/JASSS/4/2/0.html

Coleman JS (1990) Foundations of social theory. The Belknap Press of Harvard University Press, Cambridge

Föllmer H (1974) Random economies with many interacting agents. J Math Econ 1:51-62

Gordon MB, Nadal J-P, Phan D, Semeshenko V (2007) Discrete choices under social influence: generic properties. Available at http://halshs.archives-ouvertes.fr/halshs-00135405

Geels FW (2007) Transformation of large technical systems: a multilevel analysis of the Dutch highway system (1950-2000). Sci Technol Human Values 32:123-149

Geels FW, Schot J (2007) Typology of sociotechnical transition pathways. Res Policy 36:388-417

Gordon MB, Nadal J-P, Phan D, Vannimenus J (2005) Seller's dilemma due to social interactions between customers. Phys A 356:628-640

Kemp R (1998) The diffusion of biological waste-water treatment plants in the Dutch food and beverage industry. Environ Res Econ 12:113-136

Kittel CH, Kroemer H (1980) Thermal physics, 2nd edn. Freeman, New York

Lindenberg SM (1992a) The method of decreasing abstraction. In: Coleman JS, Fararo TJ (eds) Rational choice theory: advocacy and critique. Sage, Newbury Park, pp 3-20

Nadal J-P, Phan D, Gordon MB, Vannimenus J (2005) Multiple equilibria in a monopoly market with heterogeneous agents and externalities. Quant Finance 5:557-568

Popper K (1992) The logic of scientific discovery, 2nd edn. Routledge, London

Weisbuch G (2000) Environment and institutions: a complex dynamical systems approach. Ecol Econ 35:381-391

Weisbuch G, Kirman AP, Herreiner DK (2000) Market organisation and trading relationships. Econ J 110:411-436

Gérard Weisbuch physicist, PhD 1967, CNRS member, Ecole Normale supérieure from 1964 to present, except when Professor in Marseille University, 1969-1985. Working in complex systems since 1978, first biological systems and more recently (1992) on socio-economic systems: Environment, markets, opinion dynamics, and firm networks.

Vincent Buskens PhD 1999, associate professor at the Department of Sociology/ICS, Utrecht University. His research interests focus on dynamics of social networks, cooperative relations, and trust. Most recent book: Social Networks and Trust. Kluwer (2002).

Luat Vuong is a doctoral student in the School for Applied Physics at Cornell University, where she studies the nonlinear propagation dynamics of high-power light pulses. After obtaining her $\mathrm{PhD}$, she will pursue research related to nanostructured solar devices at the ICFO-Institute of Photonic Sciences in Barcelona as a postdoctoral associate. 